\title{
Femtosecond Laser Drilling of Copper: Modeling and Experiment Comparison
}

\author{
Yi-Hsien Liu ${ }^{1}$, Chung-Wei Cheng ${ }^{1}$ and Jinn-Kuen Chen $^{2}$ \\ ${ }^{1}$ Department of Mechanical Engineering, National Chiao Tung University, No. 1001, Ta Hsueh Road, Hsinchu 300, Taiwan \\ E-mail: weicheng@nctu.edu.tw \\ ${ }^{2}$ Department of Mechanical and Aerospace Engineering, University of Missouri, Columbia, Missouri 65211, U.S.A.
}

\begin{abstract}
This study experimentally and theoretically investigated the ablation of copper by a femtosecond green laser pulse of wavelength $515 \mathrm{~nm}$ and pulse duration $300 \mathrm{fs}$. The copper was processed by a single laser pulse with fluences up to $789 \mathrm{~J} / \mathrm{cm}^{2}$. A modified Drude-critical point (DCP) model that considers the temperature-dependent inter-band transition, a two-temperature model (TTM), two phase change models, and a phase explosion model were adopted to obtain the temperature-dependent properties and ablation depth. The simulated ablation depths by the proposed DCP-mod method were in better agreement with the experimental results than those by the conventional DCP model.
\end{abstract}

DOI: $10.2961 / \mathrm{jlmn} .2020 .01 .2005$

Keywords: femtosecond laser; Drude-critical point model; two-temperature model

\section{Introduction}

Femtosecond laser material processing has been demonstrated as a promising method for precise micro/nano material processing due to nonlinear multi-photon absorption and minimal collateral damage [1, 2]. A great number of theoretical and experimental works on femtosecond laser-material interactions with copper have been reported in the past twenty years. In theoretical studies, many researchers have focused on using a two-temperature model (TTM) to simulate the copper surface machined by a femtosecond laser [1, 3-5]. On the other hand, most of the experimental studies have focused on the machining using a Ti-Sapphire femtosecond laser (with a wavelength of 800 $\mathrm{nm})$ at a low fluence range, i.e. $<10 \mathrm{~J} / \mathrm{cm}^{2}[6-10]$. Therefore, theoretical simulation studies have primarily confined to this fluence range [5, 11-13].

In our previous study, we performed copper ablation with a 800-nm Ti-Sapphire femtosecond laser over a high fluence regime, such as multi-pulses with a total fluence up to $408 \mathrm{~J} / \mathrm{cm}^{2}$ [14] and a single-pulse -of fluence up to 63.4 $\mathrm{J} / \mathrm{cm}^{2}$ [15]. Recently, the microdrillings of copper using a femtosecond laser with wavelengths $1030 \mathrm{~nm}$ and $515 \mathrm{~nm}$ have been reported [16]; however, the ablation depth data were not presented. To the authors' knowledge, there is no published research comparing the simulation and experimental results of the femtosecond laser ablation of copper at the green wavelength range.

In this work, a 1D TTM with a modified Drude-critical point (DCP) model that considers the temperaturedependent inter-band transition was developed. Two phase change models and a phase explosion model were adopted to simulate material ablation. The copper was processed by a single femtosecond green laser pulse with a high fluence of 193-789 J/ $\mathrm{cm}^{2}$. The experiment results were compared with the simulations obtained by the above models.
Copper material (purity $\geq 99.97 \%$ ) was machined in air by a femtosecond fiber laser (mRadian Inc.) with a second harmonic wavelength of $515 \mathrm{~nm}$, a pulse duration of 300 fs, a repetition rate of $3 \mathrm{kHz}$, and a maximum average power of $1 \mathrm{~W}$. The laser beam had a Gaussian profile with a diameter $2.2 \mathrm{~mm}$ at $\mathrm{e}^{-2}$. The laser beam passed through an objective lens (with a numerical aperture of 0.28) and then directed normally to the target. The diameter of the focused beam on the copper surface was calculated to be about 2.2 $\mu \mathrm{m}$. The copper was machined by translating the target sample using a positioning stage.

The microholes on the copper were performed using a single laser pulse. An acousto-optic modulator (AOM) was adopted to ensure the single shot pulse. The laser powers applied were $11 \sim 45 \mathrm{~mW}$ at a repetition rate of $3 \mathrm{kHz}$. The laser fluences were calculated to be in the range of $193 \sim 789$ $\mathrm{J} / \mathrm{cm}^{2}$. After the experiment, the crater shapes were measured using a white light interference microscopy (Polytec MSA-500), and the scanning electron microscopy (SEM, Hitachi SU-8010) was used to image the top view of the crater profile.

\section{Modeling}

To accurately simulate the laser ablation of metal (copper) at a high laser fluence, a set of high fidelity optical and thermal models were needed. The surface reflectivity and absorption coefficient determine the laser energy absorption and the distribution inside the material that could significantly affect the result of the thermal ablation.

\subsection{Dynamic optical properties}

Constant optical properties, i.e., the surface reflectivity and absorption coefficient at room temperature, are often used for laser energy absorption and thermal ablation modeling. However, optical properties will change dynamically under ultra-fast laser irradiation. Using constant values could cause inaccuracy in the model predication. Recently, the ultrafast laser processing of metal (copper) with dynamic optical properties has been

\section{Experiment}


investigated both theoretically [17] and experimentally [18]. It has been shown that a DCP model with three Lorentzian terms can accurately characterize the dynamic optical properties of copper for a wide range of laser wavelengths (200-1200 nm). The DCP model with three Lorentzian terms is given as [17]:

$$
\begin{aligned}
& \epsilon(\omega)=\epsilon_{\infty}-\frac{\omega_{p}^{2}}{\omega_{L}\left(\omega_{L}+i v_{e f f}\right)}+ \\
& \sum_{p=1}^{3} B_{p} \Omega_{p}\left(\frac{e^{i \emptyset_{p}}}{\Omega_{p}-\omega-i \Gamma_{p}}+\frac{e^{-i \emptyset_{p}}}{\Omega_{p}+\omega+i \Gamma_{p}}\right) \\
& =\epsilon_{1}+i \epsilon_{2}
\end{aligned}
$$

where, $\epsilon_{\infty}$ is the dielectric constant, $\omega_{L}$ is the laser frequency, $\omega_{p}$ is the plasma frequency, $v_{\text {eff }}$ is the effective collision frequency for electrons, $B$ is a weighting factor for critical points, $\phi$ is the phase, $\Omega_{p}$ is the critical frequency (gap energy) related to the interband transitions, and $\Gamma_{p}$ is the boarding parameter of the $p^{\text {th }}$ oscillator.

In Eq. (1), the effective collision frequency for the electrons is expressed as:

$$
\begin{array}{r}
v_{e f f}=v_{e, e}\left(T_{e}\right)+v_{e, p h}\left(T_{e}, T_{l}\right) \\
=A_{e} T_{e}^{2}+1.4144 v_{e, p}
\end{array}
$$

where, $T_{e}$ and $T_{l}$ are the electron and lattice temperatures, $v_{e, e}\left(T_{e}\right)$ is the collision rate between electrons, and $v_{e, p h}\left(T_{e}, T_{l}\right)$ is the collision rate between electrons and phonons. For copper, $A_{e}$ is $1.28 \times 10^{7} \mathrm{~s}^{-1} \mathrm{~K}^{-2}$ [19] and $v_{e, p h}\left(T_{e}, T_{l}\right)$ is dependent on both $T_{e}$ and $T_{l}$ temperature and is calculated by the model proposed in [20]. The details of the above parameters in the DCP model with three Lorentzian terms and $v_{e, p}$ for copper can be found in reference [17]. Figure 1 shows the calculated values of the $\epsilon_{1}$ and $\epsilon_{2}$ parts of copper at room temperature $\left(T_{e}=T_{l}=300\right.$ $\mathrm{K})$, and the results are consistent with the theoretical values [21].

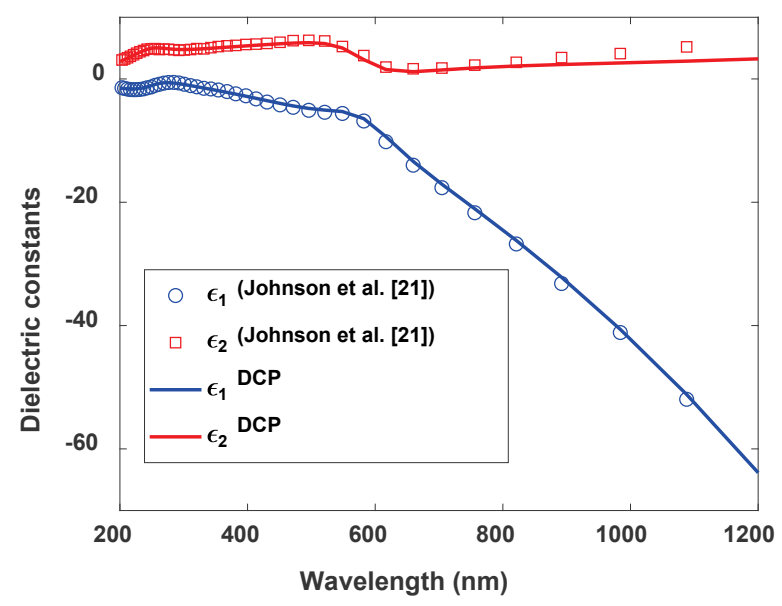

Fig. 1 Relative dielectric constant in the optical parameters of copper

With the permittivity $\epsilon_{1}$ and $\epsilon_{2}$ calculated from Eq. (1), the refractive index $(n)$ and extinction coefficient $(k)$ can be obtained by:

$$
\begin{aligned}
& n(z, t)=\sqrt{\frac{\sqrt{\varepsilon_{1}^{2}+\varepsilon_{2}^{2}}+\varepsilon_{1}}{2}} \\
& k(z, t)=\sqrt{\frac{\sqrt{\varepsilon_{1}^{2}+\varepsilon_{2}^{2}}-\varepsilon_{1}}{2}}
\end{aligned}
$$

Assume the laser beam has a perpendicular incidence to the material; the optical reflectivity $(R)$ and absorption coefficient $(\alpha)$ can be further calculated by the Fresnel functions:

$$
\begin{aligned}
& R(z, t)=\frac{(n(z, t)-1)^{2}+k^{2}(z, t)}{(n(z, t)+1)^{2}+k^{2}(z, t)} \\
& \alpha(z, t)=\frac{2 \omega_{L} k(z, t)}{c}
\end{aligned}
$$

where $c$ is the speed of light in a vacuum.

Recently, a comparative study of the experimental and simulated transient optical properties of the femtosecond laser $(528 \mathrm{~nm}, 680 \mathrm{fs})$ processing of copper was performed by Winter et al. [22]. The results showed that the experimental results cannot be compared with the theoretical calculations if the DCP model (Eq. (1)) does not consider the temperature-dependent inter-band transition.

In this study, the DCP model of Eq. (1) was modified and renamed as DCP-mod to consider the inter-band transition effect. The laser wavelength used in this study was $515 \mathrm{~nm}$, which was near the first and second critical points, and the constant boarding parameters $\Gamma_{1}$ and $\Gamma_{2}$ were replaced with a temperature-dependent parameter, i.e., $\Gamma_{1}\left(T_{e}, T_{l}\right)=A_{1} v_{e f f}$ and $\Gamma_{2}\left(T_{e}, T_{l}\right)=\Gamma_{2}+A_{2} v_{\text {eff }}$. The constant parameters $A_{1}=10.62$ and $A_{2}=1.5$ were used in this study.

Figure 2 shows the dynamic optical properties of the copper irradiated by a femtosecond laser $(528 \mathrm{~nm}, 680 \mathrm{fs})$ with laser fluences of $0.4 \mathrm{~J} / \mathrm{cm}^{2}$. In the simulation, the Gaussian pulse started from $-2 t_{p}$, reached its peak value at $t$ $=0$, and ended at $2 t_{p}$, in which $t_{p}$ is the laser pulse duration. The results of the DCP-mod were similar to the experiment results by femtosecond laser $(528 \mathrm{~nm})$ [22]. As shown in Fig. 2 , the simulated reflectivity increases quickly, reaches the peak value of 0.73 at time $-0.48 \mathrm{ps}$, decreases to the minimum of 0.47 at time $0.48 \mathrm{ps}$, and then increases at a slower rate as the time prolongs. Of note, at the time 0 ps to $0.48 \mathrm{ps}$, the minimum value of the experiment $(\sim 0.58)$ is higher than that of the simulation by DCP-mod. The discrepancy between the calculation and the experiment might be attributed to the constant plasma frequency $\left(\omega_{p}\right)$ that is used in the current model.

However, the surface reflectivity predicted by the conventional DCP decreases quickly during the laser pulse and then increases at a slower rate as time increases, which is similar to the experiment results using a femtosecond laser $(806 \mathrm{~nm})$ [23]. According to the result shown in Fig. 2, the conventional DCP model that does not consider the temperature-dependent inter-band transition was not suitable for modeling copper processing using a laser wavelength in the visible range. 


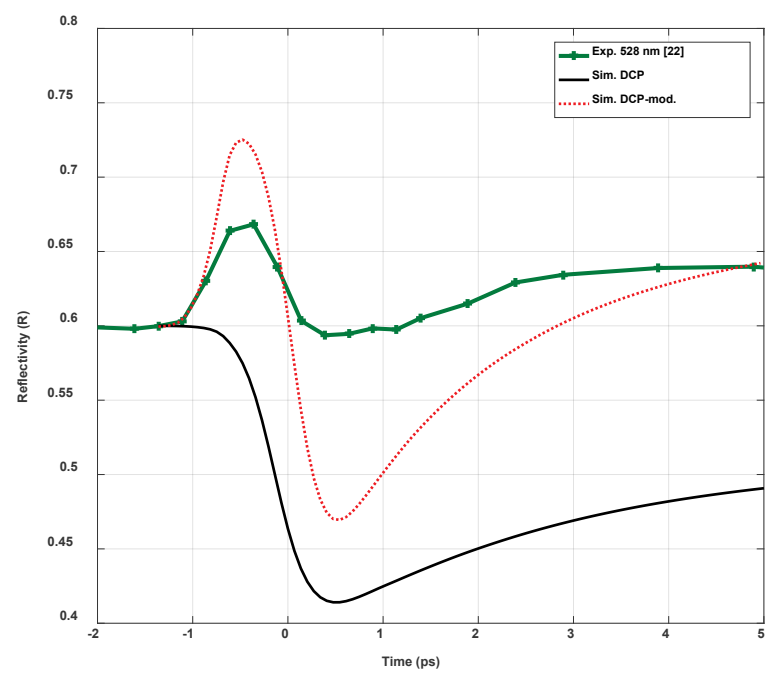

Fig. 2 Time dependence of the calculated optical properties of copper irradiated by a femtosecond laser $(528 \mathrm{~nm}, 680$

fs) and laser fluences of $0.4 \mathrm{~J} / \mathrm{cm}^{2}$

\subsection{Two temperature model}

The femtosecond laser interaction with metals only lasts for a very short period, which is less than the electron and lattice coupling time (about tens of ps) [24]. Therefore, a TTM is widely used to describe the interactions between the laser and electrons and between the electron and lattice subsystems.

Bulk copper is normally irradiated by a femtosecond laser pulse on the front surface $(z=0)$ and the laser beam propagates along the $z$-axis. The 1-D TTM is given by [25, 26]:

$$
\begin{aligned}
C_{e} \frac{\partial T_{e}}{\partial_{t}} & =\frac{\partial}{\partial_{z}}\left(K_{e} \frac{\partial T_{e}}{\partial_{z}}\right)-G\left(T_{e}-T_{l}\right)+S(z, t) \\
C_{l} \frac{\partial T_{l}}{\partial_{t}} & =\frac{\partial}{\partial_{z}}\left(K_{l} \frac{\partial T_{l}}{\partial_{z}}\right)+G\left(T_{e}-T_{l}\right)
\end{aligned}
$$

where, $T, C$ and $k$ are the temperature, heat capacities and the thermal conductivity of the electrons and lattices as denoted by $e$ and $l$. $S(z, t)$ is the laser heat source term; $G$ is the electro-phonon coupling factor. $S(z, t)$

The heat density $S$ can be expressed as [14]:

$$
\begin{aligned}
& =\frac{[1-R(0, t)] F}{1.06 t_{p}\left(\delta_{\alpha}(z, t)+\delta_{b}\right)} \exp \left[\int_{0}^{z} \frac{1}{\delta_{\alpha}(z, t)+\delta_{b}} d z\right. \\
& \left.-2.77\left(\frac{t}{t_{p}}\right)^{2}\right]
\end{aligned}
$$

where, $t_{p}$ is the full width half maximum (FWHM) of the Gaussian temporal pulse, $F$ is the laser fluence, $R(0, t)$ is the temperature-dependent surface reflectivity at $z=0, \delta_{\alpha}(z$, $t)=1 / \alpha(z, t)$ is the temperature-dependent optical penetration depth, and $\delta_{b}$ is the ballistic range. The Gaussian pulse starts from $-2 t_{p}$, reaches its peak value at $t=0$, and ends at $2 t_{p}$.

The ballistic range $\delta_{b}$ was adopted to consider the effects of the electron ballistic motion that spreads the absorbed laser energy deeper into the electrons [4, 27]. In this work, a temperature-dependent value determined by $\delta_{b}=v_{e} \times t_{b}$ was used, in which $v_{e}=\sqrt{\frac{3 k_{B} T_{e}}{m_{e}}}$ is the electron velocity, $t_{b}$ is the Drude relaxation time (e.g. $t_{b}=27 \mathrm{fs}$ [28]), $k_{B}$ is the Boltzmann constant, and $m_{e}$ is the mass of an electron.

\subsection{Phase explosion model}

During the ablation of metal by femtosecond laser pulses at high laser fluences, phase explosion is a dominating mechanism [29]. When a metal is superheated to near the thermodynamic equilibrium critical temperature $\left(T_{t c}\right)$, the mixture of vapor and liquid droplets are ejected from the material immediately. In numerical analyses of pulsed laser ablation, phase explosion is defined as occurring when the liquid temperature reaches $0.9 T_{t c}$ [30]. The details of these models can be found in references [31, $32]$. For the sake of brevity, they are not described here.

\subsection{Numerical method}

A finite difference method (tri-diagonal matrix method) was used to solve the $T_{e}$ from Eq. (7). After that, the $T_{l}$ was solved from Eq. (8). In the numerical simulation, copper with an initial temperature of $300 \mathrm{~K}$ was irradiated by a laser pulse of wavelength $515 \mathrm{~nm}$ and pulse duration $300 \mathrm{fs}$. The $T_{t c}$ was $7,696 \mathrm{~K}$ for copper. The temperature-dependent thermophysical properties can be found in references [15], and other parameters can be found in Table II in reference [33].

A total of 2500 control volumes per $\mu \mathrm{m}$ were modeled over the 1D computational domains varying from $3 \mu \mathrm{m}$ to $10 \mu \mathrm{m}$, depending on the laser fluence. When the calculated lattice temperature at a grid point reached $0.9 T_{t c}(6926 \mathrm{~K})$, that grid point was then removed from the model.

\section{Results and discussion}

\subsection{Experimental results}

Figure 3 presents the top view SEM images of the copper structures fabricated by the single femtosecond laser pulse $(515 \mathrm{~nm}, 300 \mathrm{fs})$ for different fluences $(32,193$, and $789 \mathrm{~J} / \mathrm{cm}^{2}$ ), respectively. As can be seen in Figure 3(a), the resulting textured microstructures (marked by the yellow arrow) were composed of micro bumps and cavities. The reason for the structure's formation was presumed to be the influence of the temperature gradient by the Gaussian beam. The interested readers may refer to reference [34] for more details. The microstructures in the center of the irradiated area diminished as the laser fluence was increased and a crater eventually appeared at higher fluences (for example, $193 \mathrm{~J} / \mathrm{cm}^{2}$ and $798 \mathrm{~J} / \mathrm{cm}^{2}$ ). As expected, the diameter of the crater increased as the laser fluence increased. The measured crater diameters were in the range of $14 \mu \mathrm{m}$ to $21 \mu \mathrm{m}$. It was found that at high laser fluences, such as those shown in Figure 3(c), the surrounding area of the crater had a trace of solidified molten material (a few $\mu \mathrm{m})$.

Figure 4 shows the cross-section profile of the craters for a laser fluence of 193,456 , and $789 \mathrm{~J} / \mathrm{cm}^{2}$, respectively. It appears that the depth of the craters increased as the laser fluence increased. The measured ablation depths were in the range of $2.5 \mu \mathrm{m}$ to $4 \mu \mathrm{m}$. 

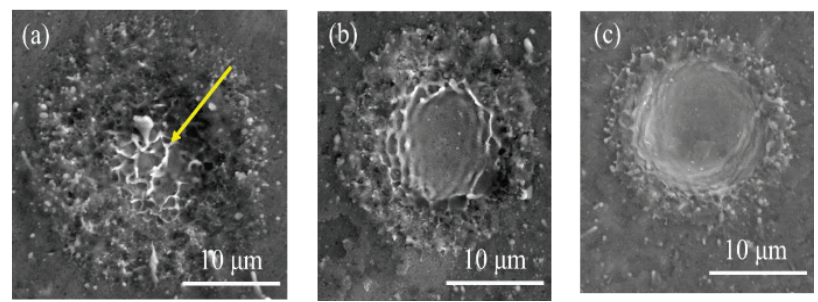

Fig. 3 SEM images of craters on copper caused by a single laser pulse with laser fluences of 32, 193 and $789 \mathrm{~J} / \mathrm{cm}^{2}$

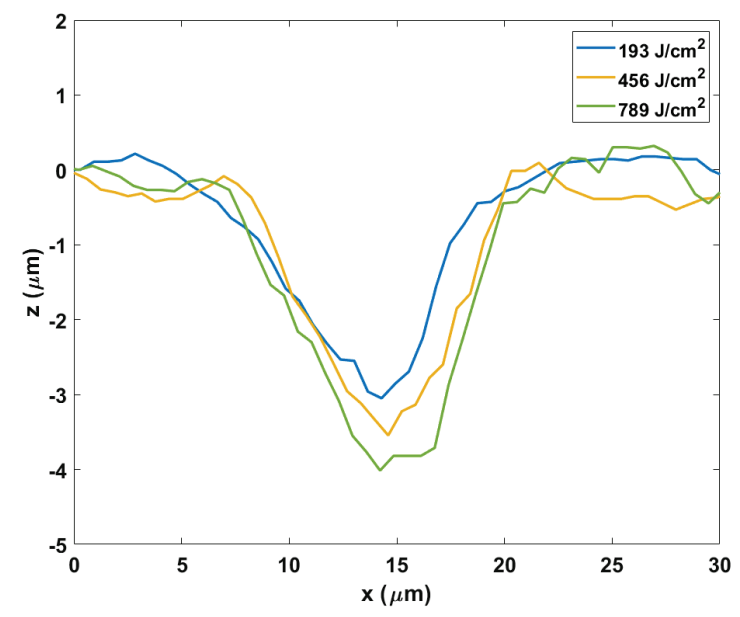

Fig. 4 Cross-section profile data

\subsection{Simulation results}

Figure 5 shows the calculated optical properties irradiated by laser fluences of $0.4,1.0$, and $193 \mathrm{~J} / \mathrm{cm}^{2}$, respectively. The normalized Gaussian temporal pulse is also presented in the figures for discussion. The Gaussian pulse started from

$-0.6 \mathrm{ps}$, reached its peak value at $t=0$, and ended at $0.6 \mathrm{ps}$. The surface reflectivity $(R)$ and the absorption coefficient $(\alpha)$ at room temperature were 0.59 and $52 \mu \mathrm{m}^{-1}$, respectively.

The simulation phenomenon of surface reflectivity was similar to that shown in Figure 2 (DCP-mod and experiment). As the laser fluence increased, the peak reflectivity became earlier and then decreased to a lower value after rising. For example, at a laser fluence of $193 \mathrm{~J} / \mathrm{cm}^{2}$, the reflectivity dropped to 0.12 at time -0.3 ps. As shown in Figure 5(b), the absorption coefficient decreased and then increased at a much slower rate as the time increased. As the laser fluence increased, the absorption coefficient decreased quickly and further. The decrease in reflectivity resulted in more laser energy deposited into the material, and the decrease in absorption coefficient caused a deeper laser penetration. It was evident that these significant changes of the optical properties influenced the amount of laser energy absorption and distribution during the femtosecond laser-material interaction, and subsequently altered the amount of material thermal ablation.

Figure 6 shows the calculated $T_{e}$ and $T_{l}$ temperatures of the copper irradiated by the three laser pulses of fluence 0.4 , 1.0 , and $193 \mathrm{~J} / \mathrm{cm}^{2}$, respectively. The smooth evolutions of the $T_{e}$ and $T_{l}$ temperatures with time for the lowest laser fluence $0.4 \mathrm{~J} / \mathrm{cm}^{2}$ were the same as most previously-reported simulation results. In this case, the lattice heated up via the electron-phonon collisions within a few picoseconds, and $T_{e}$ was equal to $T_{l}$ at about 8 ps and became smaller. The two temperatures would eventually reach the equilibrium. The maximum $T_{l}$ was $5,456 \mathrm{~K}$, which was less than $6,926 \mathrm{~K}(0.9$ $T_{t c}$ ); thus, no phase explosion occurred. For the fluences (1.0, and $193 \mathrm{~J} / \mathrm{cm}^{2}$ ) higher than the ablation threshold, the maximum $T_{l}$ remained at around $6,926 \mathrm{~K}$, which was attributed to the phase explosion.

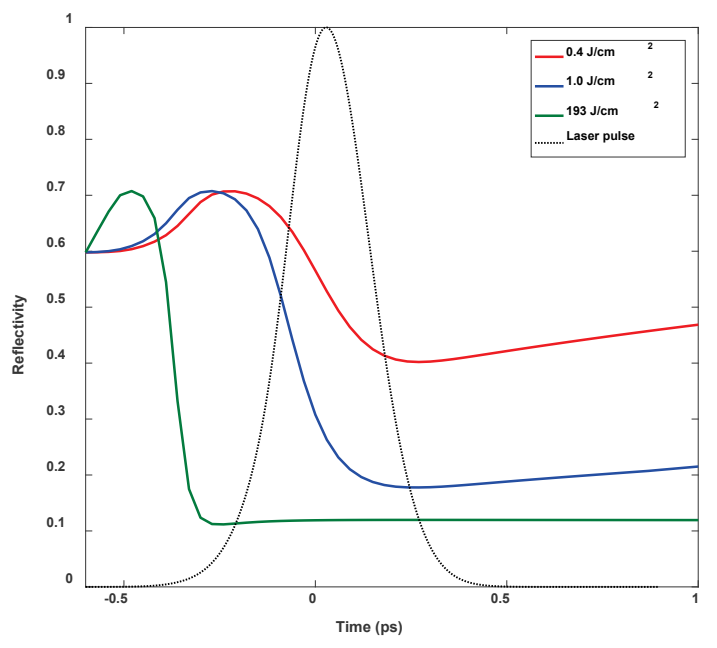

(a)

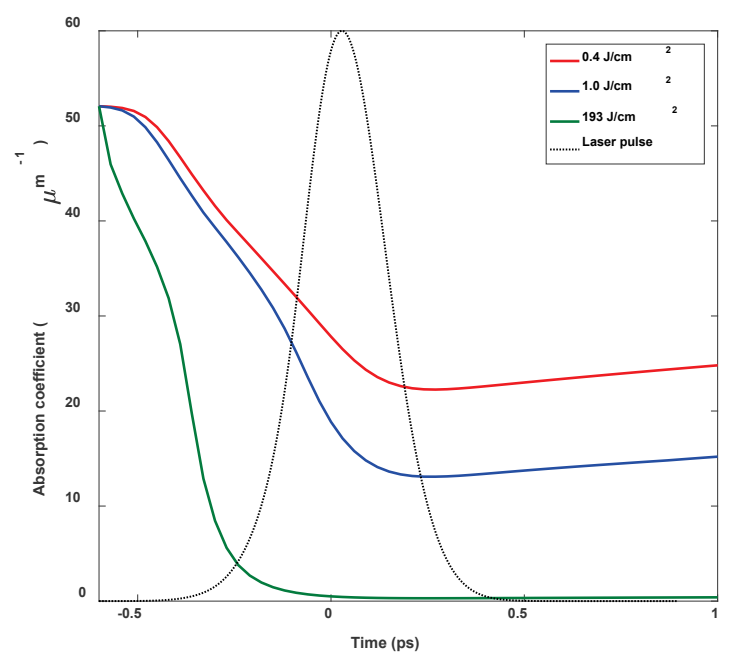

(b)

Fig. 5 Calculated optical properties: (a) surface reflectivity

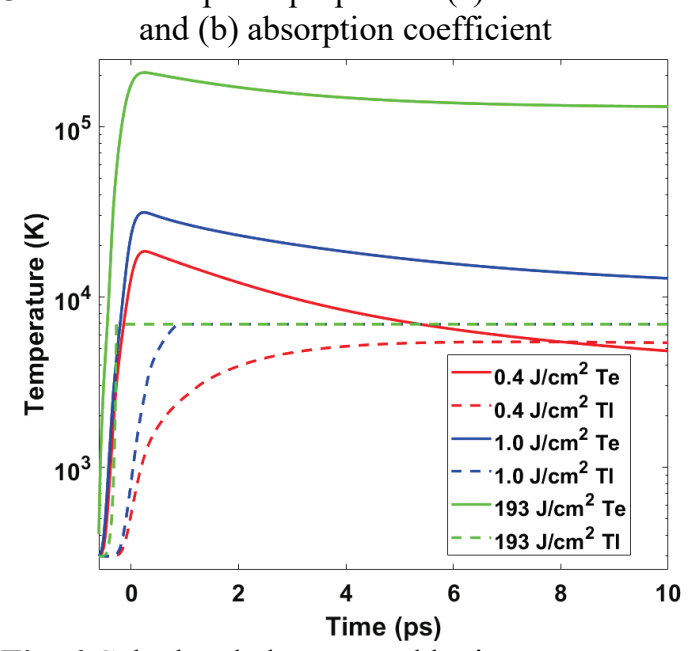

Fig. 6 Calculated electron and lattice temperature 


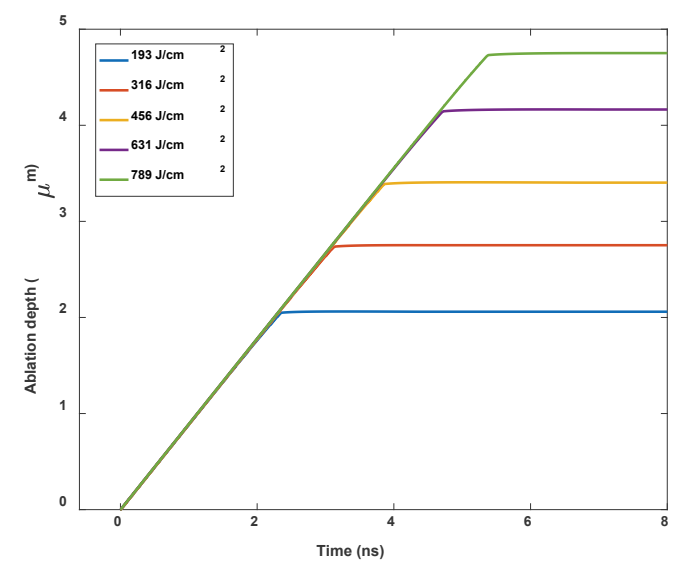

(a)

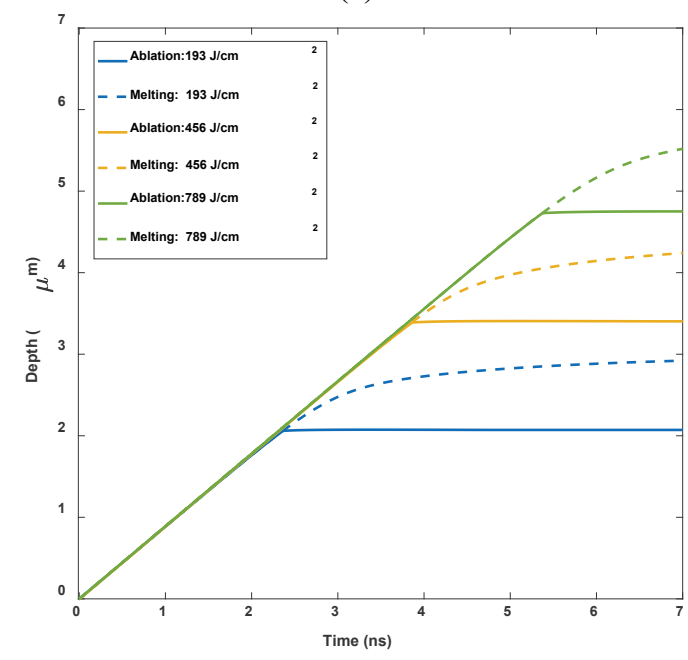

(b)

Fig. 7(a) calculated ablation depth by different fluences; (b) ablation and melting depth

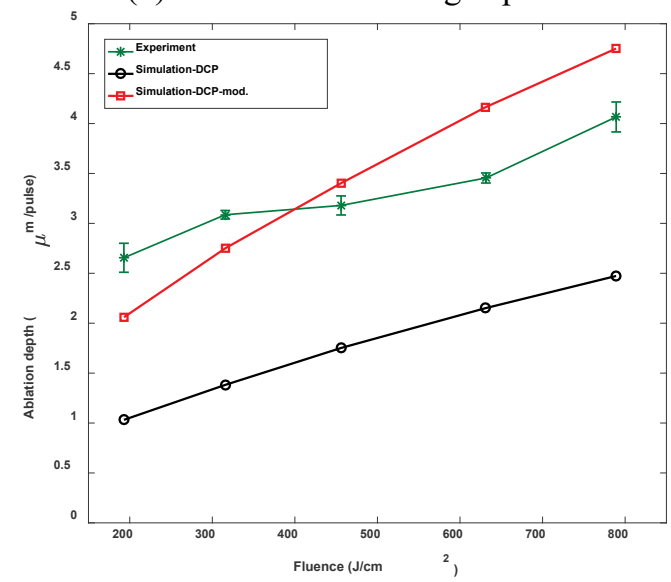

Fig. 8 Simulation and experimental ablation depth

The time history of the laser material ablation and melting are shown in Figure 7 for three different fluences. As seen in Figure 7(a), the steeper part of the material ablation was caused by the phase explosion, while the flatter part was caused by vaporization alone. It is shown here that the former is the dominating material ablation mechanism while the later can contribute some. . Figure 7(b) shows the ablation and melting depths. It was found that a thicker liquid layer was generated after the end of the phase explosion (for example, about $848 \mathrm{~nm}$ for the laser fluence $789 \mathrm{~J} / \mathrm{cm}^{2}$ at $7 \mathrm{~ns}$ ).

Figure 8 compares the ablation depth between the experimental measurements and simulation results. It can be seen that the simulations using the proposed DCP-mod method for the optical properties were in good agreement with the experimental data over a wide range of laser fluences. However, the simulations by the conventional DCP model (without considering the inter-band transition effect) significantly underestimated the ablation depth. It was found that in the high fluence $\left(>500 \mathrm{~J} / \mathrm{cm}^{2}\right)$ regime, the simulated ablation depth was higher than the experimental data. There were two reasons which could explain this situation. First of all, the peak power intensity used in this study was in the range from $6.4 \times 10^{14} \mathrm{~W} / \mathrm{cm}^{2}$ to $2.6 \times 10^{15}$ $\mathrm{W} / \mathrm{cm}^{2}$, which was higher than the air breakdown threshold (i.e. $2.4 \times 10^{14} \mathrm{~W} / \mathrm{cm}^{2}$ ). The laser fluence loss due to air breakdown is $10 \%$ to $25 \%$ as the intensity increased [35]. However, in this study, this situation wasn't considered on the simulation so that the simulation value was overestimated in the high fluence regime.

The second reason was speculated that the focused beam diameter (about $2.2 \mu \mathrm{m}$ ) would be similar to the size of the heat-affected zone (few $\mu \mathrm{m}$ ) and thus, the use of the simplified 1D TTM approach could not be sufficient to neglect the loss of lateral heat conduction. In order to more accurately predict the material ablation behavior, in addition to the dynamic optical model proposed in this study, a 2D axisymmetric TTM model needs to be adopted.

\section{Conclusion}

In this study, experimental and theoretical results were reported for the single femtosecond laser pulse ablation of copper at fluences up to $789 \mathrm{~J} / \mathrm{cm}^{2}$. The laser ablation experiments were performed with a femtosecond fiber laser pulse of wavelength $515 \mathrm{~nm}$ and duration $300 \mathrm{fs}$. Numerical simulations were carried out using the predictive optical and thermal models, including a modified DCP model that considered the temperature-dependent inter-band transition, two-temperature model, two phase change models, and the phase explosion model for the ablation phenomenon. It was shown that the simulations by the proposed DCP-mod method were in good agreement with the experimental data over a wide range of laser fluences and that simulations by the conventional DCP model would underestimate the ablation depth.

\section{Acknowledgements}

The authors gratefully acknowledge the support of the Ministry of Science and Technology for this research under grants MOST 107-2221-E-009-061-MY3 and the femtosecond laser support from mRadian Co. Ltd

\section{References}

[1] S. Nolte, C. Momma, H. Jacobs, A. Tunnermann, B.N. Chichkov, B. Wellegehausen, and H. Welling: J. Opt. Soc. Am. B.,14. (1997), 2716

[2] R.R. Gattass and E. Mazur: Nat. Photocics., 2. (2008), 219

[3] J. Yang, Y. Zhao, and X. Zhu: Appl. Phys. A. Mater., 89. (2007), 571

[4] J. Byskov-Nielsen, J.-M. Savolainen, M.S. Christensen, 
and P. Balling: Appl. Phys. A. Mater., 103. (2011), 447

[5] B. Wu and Y.C. Shin: Appl. Surf. Sci., 253. (2007), 4079

[6] M. Hashida, A.F. Semerok, O. Gobert, G. Petite, Y. Izawa, and J.F. Wagner: Appl. Surf. Sci., 197. (2002), 862

[7] J. Byskov-Nielsen, J.M. Savolainen, M.S. Christensen, and P. Balling: Appl. Phys. A. Mater., 101. (2010), 97

[8] Y. Miyasaka, M. Hashida, T. Nishii, S. Inoue, and S. Sakabe: Appl. Phys. Lett., 106. (2015), 013101

[9] R. Le Harzic, D. Breitling, M. Weikert, S. Sommer, C. Fohl, S. Valette, C. Donnet, E. Audouard, and F. Dausinger: Appl. Surf. Sci.,249. (2005), 322

[10] R. Le Harzic, D. Breitling, M. Weikert, S. Sommer, C. Fohl, S. Valette, C. Donnet, E. Audouard, and F. Dausinger: Appl. Surf. Sci., 249. (2005),

[11] J. Yang, Y. Zhao, and X. Zhu: Appl. Phys. A.,89. (2007), 571

[12] A. Suslova, A. Elsied, and A. Hassanein: Laser. Part. Beams.,36. (2018), 144

[13] S. Li, S. Li, F. Zhang, D. Tian, H. Li, D. Liu, Y. Jiang, A. Chen, and M. Jin: Appl. Surf. Sci., 355. (2015), 681

[14] S.Y. Wang, Y. Ren, C.W. Cheng, J.K. Chen, and D.Y. Tzou: Appl. Surf. Sci., 265. (2013), 302

[15] C.W. Cheng, S.Y. Wang, K.P. Chang, and J.K. Chen: Appl Surf Sci., 361. (2016), 41

[16] S. Döring, A. Ancona, S. Hädrich, J. Limpert, S. Nolte, and A. Tünnermann: Appl. Phys. A, 100. (2010), 53

[17] Y. Ren, J. Chen, and Y. Zhang: Jpn. J. Appl. Phys., 10. (2011), 113102

[18] J. Winter, S. Rapp, M. Schmidt, and H.P. Huber: Appl Surf Sci., 417. (2017), 2

[19] K. Vestentoft and P. Balling: Appl. Phys. A. Mater., 84. (2006), 207

[20] D. Fisher, M. Fraenkel, Z. Henis, E. Moshe, and S. Eliezer: Phys. Rev. E., 65. (2002),

[21] R. https://refractiveindex.info/?shelf $=$ main $\&$ book $=C u \& p$ age $=$ Johnson.

[22] J. Winter, S. Rapp, M. Schmidt, and H.P. Huber, Ultrafast laser energy deposition in copper revealed by simulation and experimental determination of optical properties with pump-probe ellipsometry (SPIE LASE). SPIE, 2017.

[23] A.S. Sandhu, A.K. Dharmadhikari, and G.R. Kumar: Jpn. J. Appl. Phys., 97. (2005), 023526

[24] B.I. Cho, T. Ogitsu, K. Engelhorn, A. Correa, Y. Ping, J. Lee, L.J. Bae, D. Prendergast, R. Falcone, and P. Heimann: Sci. Rep. UK., 6. (2016), 18843

[25] S.I. Anisimov, B.L. Kapeliovich, and T.L. Perel'man: J Exp Theor Phys, 39. (1974), 375

[26] J.K. Chen and J.E. Beraun: Numer. Heat. Tr. A. Appl., 40. (2001), 1

[27] V. Schmidt, W. Husinsky, and G. Betz: Phys. Rev. Lett., 85. (2000), 3516

[28] K.M. Yoo, X.M. Zhao, M. Siddique, R.R. Alfano, D.P. Osterman, M. Radparvar, and J. Cunniff: Appl. Phys. Lett., 56. (1990), 1908

[29] D.A. Willis and X. Xu: Int. J. Heat. Mass. Tran., 45. (2002), 3911

[30] R. Kelly and A. Miotello: Appl. Surf. Sci., 96-98. (1996), 205
[31] J. Huang, Y. Zhang, and J.K. Chen: Appl. Phys. A, 103. (2011), 113

[32] Y. Ren, J.K. Chen, and Y. Zhang: Int. J. Heat. Mass. Tran., 55. (2012), 1260

[33] Y. Ren, J.K. Chen, and Y. Zhang: Jpn. J. Appl. Phys., 110. (2011),

[34] E. Gurevich: Phys. Rev. E., 83. (2011), 031604

[35] W. Hu, Y.C. Shin, and G. King: Appl. Phys. Lett., 99. (2011), 234104

(Received: September 4, 2019, Accepted: February 23, 2020) 